\title{
Perforation during underwater EMR
}

\author{
Prasanna L. Ponugoti, MD, Douglas K. Rex, MD
}

Indiana University School of Medicine, Division of Gastroenterology and Hepatology Indianapolis, Indiana, USA

Underwater EMR is effective and safe and does not require submucosal injection. Here, we describe the first perforation during underwater EMR.

A 62-year-old man had a 30-mm flat lesion in the proximal ascending colon visible only in retroflexion. It appeared that submucosal injection would move it too close to the colonoscope tip to permit easy therapy. During underwater EMR, transection of the first piece resulted in perforation (Fig. A), which was closed with 3 clips (Cook Endoscopy, Winston-Salem, NC; Fig. B). CT confirmed free air without fluid collections.

The patient remained asymptomatic (he was afebrile and his white blood cell count was normal). Because the EMR was not completed, right hemicolectomy was performed 30 hours later. The perforation was adjacent to pericolonic fat, without peritoneal contamination. After primary anastomosis, the patient's recovery was uneventful. D.K.R. performed $>30$ underwater EMRs of lesions $>2 \mathrm{~cm}$ before and $>20$ after this perforation, all without adverse events. We believe this perforation was caused by the retroflexed instrument stretching the colon wall, preventing the lesion from floating. Fortunately, water did not enter the peritoneal cavity. Caution is appropriate when performing underwater EMR in retroflexion. Colonoscopists performing underwater EMR should look for the lesion floating as a safety predictor.

\section{Disclosure}

Dr Rex received research support from, and is a consultant for, Olympus America Corporation and Boston Scientific. All other authors disclosed no financial relationships relevant to this publication.

This is the author's manuscript of the article published in final edited form as:

Ponugoti, P. L., \& Rex, D. K. (2016). Perforation during underwater EMR. Gastrointestinal Endoscopy, 84(3), 543544. http://doi.org/10.1016/j.gie.2016.01.021 


\section{Commentary}

EMR is a surgery-sparing technique for noninvasive colorectal neoplasia. Over the last decade, injection-assisted EMR has gained wide acceptance and has become a technique of choice for most colorectal sessile and nonpolypoid neoplastic lesions. Injection-assisted EMR encompasses a submucosal injection of saline solution, which can be used in conjunction with epinephrine, methylene blue, and methylcellulose solutions, and results in separation of the mucosal neoplastic lesion from the underlying muscle layer (muscularis propria) creating a safety cushion. Subsequently, the neoplastic lesion can be resected safely with a snare without laceration of the muscle layer and a negative resection margin can be achieved. Successful resection of colorectal neoplasia by injection-assisted EMR can be performed in $>90 \%$ of cases, with perforation rates of $1 \%$ or less.

Underwater EMR (UEMR) was first reported by Binmoeller in 2012. In contrast to injectionassisted EMR, UEMR uses full water immersion instead of air insufflation, which results in less colonic wall distension and consequently less or no thinning of the colonic wall, whereas injection-assisted EMR could result in paradoxical widespread flattening making snare resection difficult.

The case presented here delineates another important feature of UEMR when submucosal injection results in too close proximity between the colonoscope and the neoplastic lesion. In this particular case, UEMR caused a perforation during the first attempt at snare resection. The important observation is that UEMR was performed in a retroflex position, which is often performed with locked knobs causing additional stress on the colonic wall as a result of limited endoscope mobility and subsequent colonic wall thinning. In agreement with the authors' hypothesis, this is the likely cause of the observed perforation. The lesson learned from this report is that the endoscopist needs to limit the stress on the colonic wall while performing UEMR to avoid wall thinning. If that is not possible, then alternative resection methods need to be considered, eg, using injection-assisted EMR with cap-assisted colonoscopy. 
Figure
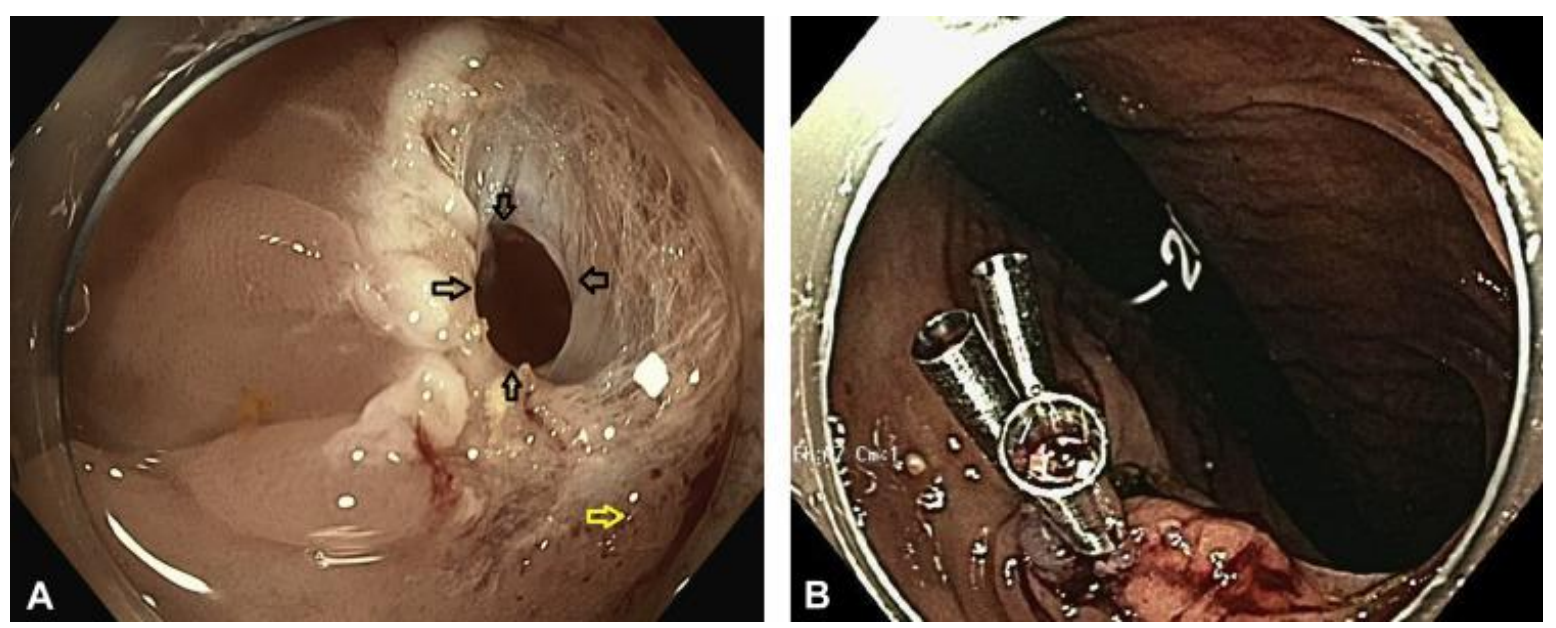